\title{
Estudio de la Cinética de Rehidratación de Zanahoria (Daucus Carota) Deshidratadas
}

Yendi I. Melquíades, Clara López y Marta E. Rosas

Universidad Nacional Autónoma de México, Facultad de Estudios Superiores Cuautitlán, Av. $1^{\circ}$ mayo s/n, Col. Atlanta, 04740 Cuautitlán Izcalli, Estado de México-México (e-mail: clara_key@hotmail.com)

\begin{abstract}
Resumen
Se presenta un estudio cinético de la rehidratación de zanahorias para dos tipos de cortes o geometrías (placas y cilindros). Las zanahorias fueron lavadas, peladas y cortadas para someterlas a un tratamiento de 5 min. en agua destilada a $80^{\circ} \mathrm{C}$ antes de ser deshidratadas en un horno de convección a diferentes temperaturas. La cinética de rehidratación se obtuvo por medio de un modelo difusional y se determinó su posible dependencia con la temperatura mediante una relación de tipo Arrhenius. Se observó que la rehidratación se ve afectada por el pretratamiento debido a los cambios estructurales, que finalmente afectan la capacidad de rehidratación de las muestras. Los coeficientes de difusión no presentan tal dependencia.
\end{abstract}

Palabras clave: rehidratación, zanahoria, coeficiente de difusión, pre-tratamiento, deshidratación

\section{Rehydration Kinetics Study of the Dehydrated Carrot (Daucus carota)}

\begin{abstract}
A kinetic study of the rehydration of carrots for two types of cuts (geometries) (slabs and cylinders), is presented. The carrots were washed, peeled and cut an then treated for $5 \mathrm{~min}$ in distilled water at $80^{\circ} \mathrm{C}$ before being dehydrated in a convection oven at different temperatures. The rehydration kinetics was obtained by means of a diffusion model and its temperature dependence was determined by an Arrhenius type relationship. It was observed that the rehydration is affected by the pretreatment due to the structural changes that finally affect the rehydration capacity of the samples. Diffusion coefficients do not present such dependence.
\end{abstract}

Keywords: rehydration, carrot, diffusion coefficient, pre-treatment, dehydration 


\section{INTRODUCCIÓN}

La apreciación de la zanahoria (Daucus carota) como producto de gran valor nutricional se debe al descubrimiento, en 1919, de que los carotenoides son un aporte de pro-vitamina $A$, la que se degrada a retinol o vitamina $A$ en el organismo humano. El producto natural, no procesado, es utilizado cocido en ensaladas frías, o en guisados, y se reduce una tendencia creciente a su uso en ensaladas crudas. En la agroindustria se le usa como materia prima para congelados, deshidratados, encurtidos, enlatados y jugos (Krarup y Moreira, 1998).

Uno de los cambios físicos que se presenta durante el secado de alimentos es la reducción del volumen así como la perdida de agua, lo que provoca que la estructura celular sufra tensiones, dando como resultado la disminución de las dimensiones y un cambio en la forma del alimento (Mayor y Sereno, 2004).

Todo pretratamiento antes del secado tiene cierta influencia sobre el producto deshidratado en el proceso posterior de rehidratación. Las características de calidad de un alimento deshidrato que ha sido rehidratado pueden mejorarse aplicando pretratamientos como: inmersión en soluciones azucaradas, salinas $(\mathrm{NaCl}) \mathrm{o}$ ácidas (ácido cítrico y/o ascórbico), escaldado, deshidratación osmótica, microondas, entre otros. Por ejemplo el escaldado, provoca cambios en las propiedades físicas del tejido (destrucción de membranas celulares) y pérdida de sólidos solubles que afectan la velocidad del secado (Marín et al., 2006; Górniki y Kaleta, 2007).

La mayoría de los productos deshidratados son generalmente rehidratados para su uso. Al rehidratar se pretende obtener productos que al reconstituirse adquieran lo más posible sus características iniciales y que lo hagan en el menor tiempo; sin embargo éste proceso se ve influido por las condiciones del secado, la preparación de la muestra, la composición de la muestra, el grado de ruptura estructural y química (McMinn y Magee, 1997). La rehidratación de materiales deshidratados está compuesta de tres procesos simultáneos: inhibición del agua respecto al material deshidratado, el hinchamiento y la lixiviación de los sólidos solubles. Antes de aplicar rehidratación a alimentos deshidratados, se deben conocer las características del alimento en su estado fresco y deshidratado, ya que las propiedades físico-químicas, mecánicas (microestructurales), sensoriales y nutricionales, cambian considerablemente de un producto fresco a deshidratado, de tal manera que estos factores determinan el comportamiento de los alimentos en el proceso de rehidratación (Krokida y MarinosKroudis, 2003; Marín et al., 2006).

El grado de rehidratación está en función del grado de ruptura de la célula y de su estructura. Krokida y Marinos-Kroudis (2003), Ramos et al. (2003) y Lewiki et al. (1997), observaron que durante el secado se presenta una ruptura celular irreversible, resultando la pérdida de la integridad y, por lo tanto, una estructura densa de vasos capilares, encogidos y destruidos debido a la reducción de las propiedades hidrófilas, que refleja una incapacidad en la retención de agua suficiente del producto rehidratado. El encogimiento origina que el volumen del producto disminuya debido a que el agua es evaporada, produciendo que la estructura superficial durante el secado se deforme fácilmente provocando huecos, mientras que la parte interna tiene pocas rupturas, donde la difusividad es reducida debido a que el agua restante está ligada por puentes de hidrogeno. Por lo tanto se espera que la microestructura de los poros y la porosidad en sí, jueguen un papel importante en el mecanismo de rehidratación (capilaridad, absorción, difusión) (Lewicki et al., 1997; Marabi y Saguy, 2004).

En cuanto a la transferencia de materia ocurrida durante la rehidratación, se puede mencionar que el agua es absorbida más rápidamente al inicio del proceso y luego disminuye gradualmente la absorción hasta que el contenido de humedad alcanza un equilibrio, es decir, que todos los espacios inter o intracelulares queden saturados con agua (Marín et al., 2006).

Existen estudios que demuestran que el proceso de adsorción puede ser considerado como un mecanismo difusional (Sanjuán et al., 1999) por lo tanto el coeficiente de difusión puede ser determinado por la segunda Ley de Fick. 
El objetivo de este trabajo fue determinar los coeficientes de difusión, así como evaluar el efecto de la temperatura, geometrías (placa y cilindro) en la cinética de rehidratación de zanahorias pretratadas y deshidratadas previamente.

\section{MODELO}

A partir de la segunda Ley de Fick (1), es posible determinar el coeficiente de difusión $\left(D_{\text {ef }}\right)$ suponiendo que el movimiento de humedad es unidimensional, continuo y homogéneo:

$$
\frac{\partial X}{\partial t}=D_{e f} \frac{\partial^{2} X}{\partial x^{2}}
$$

Para resolver esta ecuación se consideró que: (i) el contenido de humedad inicial es uniforme; (ii) la geometría se mantiene continua durante el proceso de rehidratación; (iii) la superficie consigue la humedad de saturación en un instante al estar en remojo. La condición inicial es el contenido de humedad de la muestra al principio del proceso. Asumiendo estas hipótesis, la solución del modelo fue obtenida analíticamente por el método de separación de variables, para cada geometría (Sanjuán et al., 1999); que empleando sólo el primer término de la serie solución, resulta, respectivamente:

Placa

$M=\frac{X-X_{1}}{X_{0}-X_{1}}=\frac{8}{\pi^{2}} \exp \left(\frac{-D_{e f} \pi^{2} t}{4 L^{2}}\right)$

Cilindro

$M=\frac{X-X_{1}}{X_{0}-X_{1}}=\frac{4}{5.783} \exp \left(\frac{-5.783 D_{e f} t}{r^{2}}\right)$

En la transferencia de calor, la constante de velocidad $(k)$ puede depender de la temperatura $(T)$ por una función como:

$k=k_{0} \exp \left(\frac{-E a}{R T}\right)$

Puede establecerse que en la transferencia de masa, el coeficiente de difusión se ajusta a la ecuación de Arrhenius debido a su dependencia con la temperatura (Martínez-Navarrete, 1998):

$D_{e f}=D_{0} \exp \left(\frac{-E_{a}}{R T}\right)$

Donde $D_{0}$ es un factor pre-exponencial, $E_{a}$ es la energía de activación $(\mathrm{kJ} / \mathrm{mol})$ y $R$ la constante de los gases (8,314 J/ (g-molK)) (Geankoplis, 1998).

También la razón o capacidad de rehidratación $(\mathrm{RC})$, la cual expresa la habilidad del material para rehidratarse, puede calcularse de la siguiente manera:

$R C=\frac{W r}{W d}$ 
dónde $\mathrm{Wr}$ es el peso total al final del proceso de rehidratación y Wd es el peso del material seco (Doymaz, 2008).

\section{MATERIALES Y MÉTODOS}

Se utilizaron zanahorias (Daucus carota) obtenidas en un supermercado local. Las zanahorias frescas fueron lavadas, peladas y cortadas en placas de dimensiones de $25 \mathrm{~mm} \times 19 \mathrm{~mm} \times 3 \mathrm{~mm}$ y cilindros con dimensiones de $25 \mathrm{~mm}$ de diámetro con $3 \mathrm{~mm}$ de longitud; después las muestras fueron pretratadas colocándolas en una inmersión de agua destilada a $80^{\circ} \mathrm{C}$ durante 5 minutos y enfriadas a temperatura ambiente, lo cual ablanda el tejido y logra la desgasificación, facilitando la eliminación de agua con lo que se mejora la textura, el consumo de agua y la velocidad de reconstitución durante la rehidratación (Gornicki y Kaleta, 2007).

Se secaron a 40,60 y $80{ }^{\circ} \mathrm{C}$ con una velocidad de aire constante de $0.51 \mathrm{~m} / \mathrm{s}$ en un secador de convección forzada; el secado fue realizado hasta que el cambio fuera menor de $0.2 \mathrm{~g}$ del peso inicial. Las zanahorias deshidratadas fueron guardadas en un desecador con el fin de no cambiar la humedad de la muestra.

Las muestras fueron rehidratadas sumergiéndolas en un baño de agua destilada con una proporción de 50:1 (peso muestra:peso agua) a $80^{\circ} \mathrm{C}$ (Bilbao-Sainz et al., 2005), determinando el peso en una balanza digital, OHAUS modelo As200, cada minuto hasta el término del proceso que fue de 80 minutos; el exceso de agua fue retirada colocando las muestras en una rejilla durante 10 minutos. El contenido de humedad de las muestras se determinó por el método 934.06 del AOAC (1990).

Para ajustar las curvas de las cinética de rehidratación y llegar a obtener los parámetros del modelo difusional, se aplicaron intervalos de confianza de 95\% para las curvas experimentales completas.

\section{RESULTADOS Y DISCUSIÓN}

Una vez que se colocaron las muestras secas en el agua para rehidratarlas, las paredes celulares la absorbieron, suavizándose para regresar a su forma original, debido a la elasticidad natural de la estructura celular (McMinn y Magee, 1997). Esto se puede observar en la relativamente rápida velocidad de reconstitución durante los primeros minutos, seguido por un incremento gradual que tiende a un máximo en la razón de rehidratación (RC).

Al realizar el gráfico (figuras 1 y 2) de adsorción de agua (rehidratación) se observa un primer segmento, el cual representa una tendencia lineal; puesto que la muestra deshidratada contiene moléculas que se encuentran formando puentes de hidrogeno en las regiones hidrófilas, que al estar en contacto con moléculas de agua quedan retenidas por interacciones de Van der Waals hasta saturar los poros superficiales de la muestra deshidratada, formando la fase sólida.

El segundo periodo presenta una tendencia de adsorción de humedad de manera exponencial, donde las moléculas de agua comienzan a penetrar al interior de la fase sólida, correspondiente al fenómeno de difusión, dándose un movimiento de solutos en donde se dispersa el material soluble formando la fase liquida; cuando existe agua suficiente en el alimento el sistema llega al equilibrio (Sanjuán et al., 1999). Por lo que puede ser representado por el modelo difusional de Fick (ecs. 2-3).

En ambas figuras se observa la influencia de la temperatura sobre la cinética de rehidratación, la cual fue mayor para $60^{\circ} \mathrm{C}$, y casi igual para 40 y $80^{\circ} \mathrm{C}$, lo que influye finalmente el los coeficientes de difusión. El uso del pretratamiento afecta la permeabilidad de las membranas celulares, por lo que la rapidez con la que se lleva a cabo la transferencia de agua durante el secado se incrementa conforme aumenta la temperatura, disminuyendo el tiempo y reduciendo el daño estructural con un aumento de la porosidad, facilitando la rehidratación de las muestras en un menor tiempo (Gowen et al., 2007). Sin embargo, lo que se llama porosidad abierta es la que está relacionada con el colapso de la estructura, producido durante el secado, y que impide la rehidratación (Marabi y Saguy, 2004). Investigaciones realizadas por Cunningham et al. (2008), han demostrado que el uso de un pretratamiento durante un tiempo superior a 5 minutos tiene un efecto perjudicial sobre la cinética de 
rehidratación, debido a que los carbohidratos presentes se gelatinizan totalmente lo que indica cambio en la estructura interna de las muestras aunado a una pérdida de sólidos durante rehidratación.

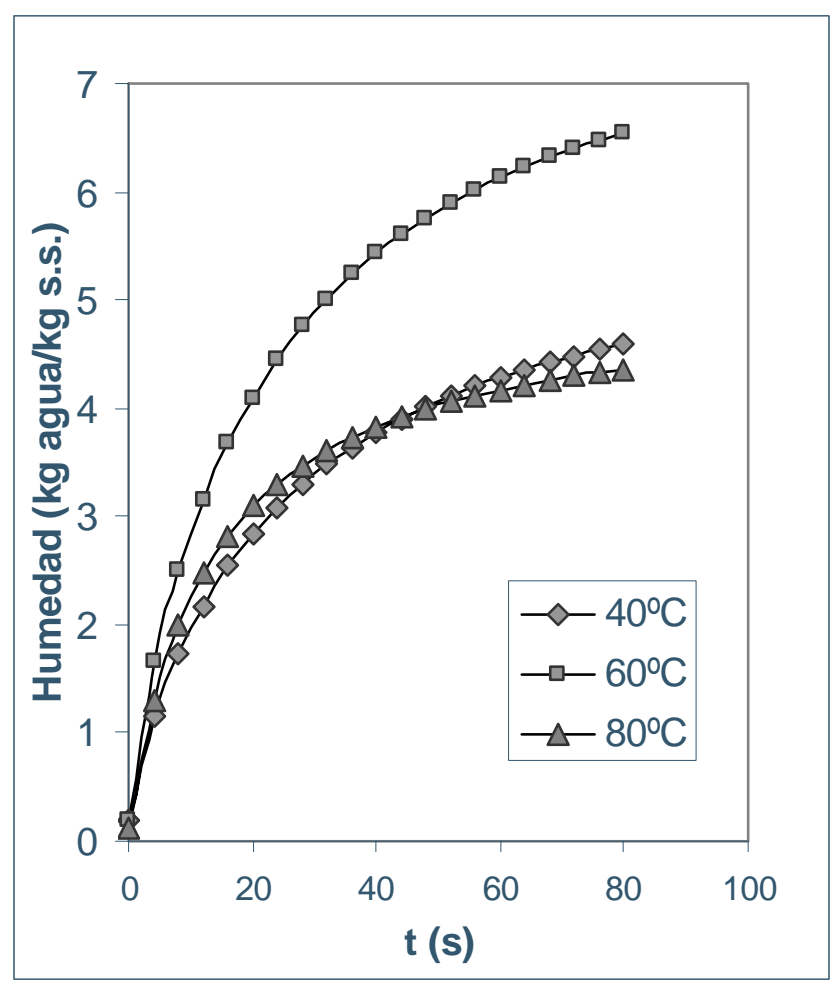

Fig. 1: Cinética de rehidratación para placas deshidratadas de zanahoria.

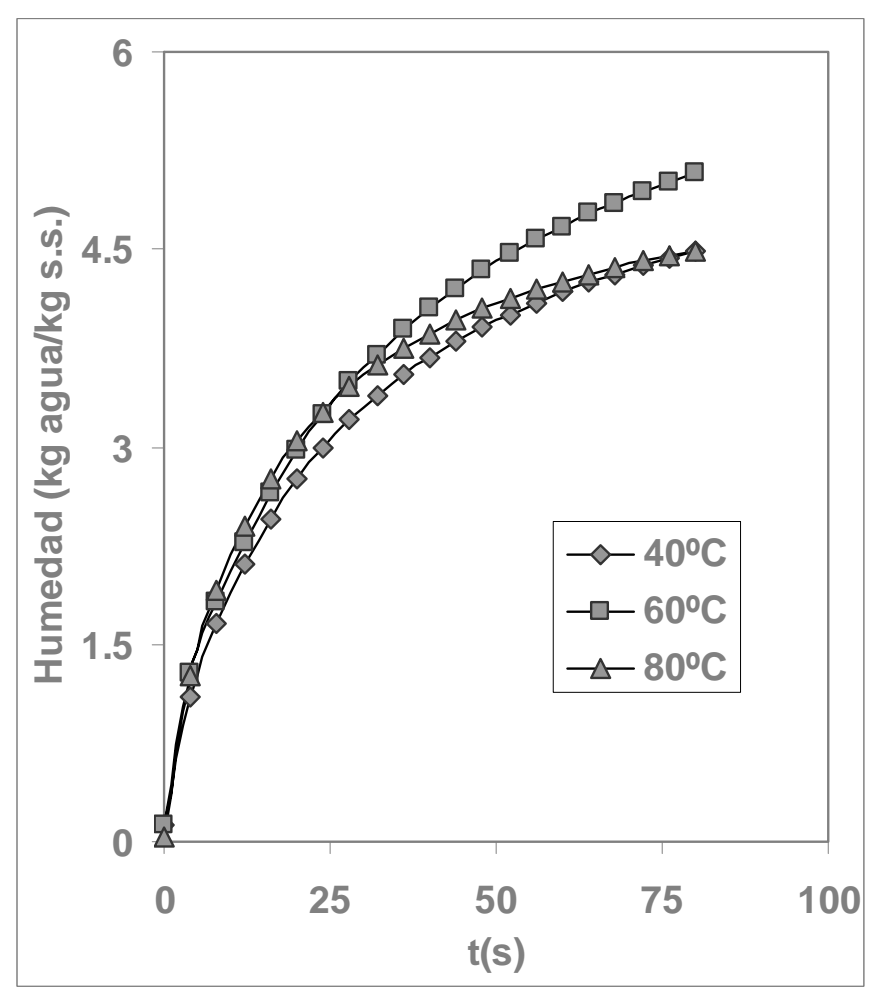

Fig. 2: Cinética de rehidratación para cilindros deshidratados de zanahoria.

Esto puede ser explicado por que durante el proceso de secado, las proteínas que forman parte de la membrana celular de la zanahoria son desnaturalizadas, produciendo que ésta se vuelva porosa e incapaz de mantener las células turgentes, por lo que al experimentar el proceso de rehidratación, las membranas de las muestras sufren defectos de acoplamiento provocando la pérdida de la integridad de la estructura. Mientras que los carbohidratos forman un sólido amorfo debido a la migración de agua, lo que permite que a medida que se lleva el proceso de secado aumenten las fuerzas cohesivas y por tanto reduce la movilidad de las moléculas (Fernández, 2006; Gornicki y Kaleta, 2007).

La capacidad de absorción de agua de productos biológicos está relacionada con el tipo de material, ultraestructura y composición de las células (García-Pascual et al., 2005). Entonces los cambios observados en el valor de RC se relacionan con los cambios químicos o físicos de la estructura sufridos por el material durante el secado, y de acuerdo a los valores obtenidos se puede decir que es dependiente de la temperatura, y por lo tanto del tiempo de proceso.

En la tabla 1 se presentan los valores de RC así como los coeficientes de difusión en rehidratación, obtenidos para cada una de las temperaturas de secado a las que fueron sometidas las muestras. Puede observarse que, en general, la difusión es menor en rehidratación comparada con los coeficientes de difusión que presentaron durante el secado $\left(2,24 \times 10^{-10} \mathrm{~m}^{2} \mathrm{~s}^{-1}\right.$ a $\left.4,78 \times 10^{-10} \mathrm{~m}^{2} \mathrm{~s}^{-1}\right)$ de las mismas. Los intervalos de humedad en los que los $D_{\text {ef }}$ fueron calculados durante la rehidratación, determinados por la pendiente de la curva en un gráfico In M vs tiempo, estuvieron entre 1.99-6.54 g/g de sólido seco; los intervalos se consideraron para dar solución de la segunda ley de Fick, para tiempos largos, con un solo término de la serie, cuando $\mathrm{M}<0.6$ (Crank, 1975).

A menor temperatura de secado mayor tiempo de proceso, por lo tanto un mayor grado de encogimiento, lo cual implica que se tienen estructuras celulares más densas y rígidas, con espacios 
intercelulares menos definidos que restringen la difusión del agua (McMinn y Magee, 1997). Esto puede observarse en los valores del coeficiente de difusión menores para $40^{\circ} \mathrm{C}$, así como en el valor de RC, donde se presume que la estructura tenía mayor daño y menor capacidad de rehidratación. También la menor humedad de equilibrio alcanzada en la rehidratación, es indicativa de los cambios en el producto, inducidos por las temperaturas y/o tiempos de secado (40 y $80^{\circ} \mathrm{c}$ ), causando menor permeabilidad dentro de la estructura y pérdida de sólidos durante la rehidratación (Cunningham et al., 2008).

Tabla 1: Coeficientes de difusión para rehidratación de zanahoria.

\begin{tabular}{|c|c|c|c|c|}
\hline $\mathrm{T}\left({ }^{\circ} \mathrm{C}\right)$ & $\begin{array}{c}\mathrm{D}_{\text {ef cilindro }} \\
\left(\mathrm{m}^{2} / \mathrm{s}\right)\end{array}$ & $\mathrm{RC}$ & $\begin{array}{c}\mathrm{D}_{\text {ef placa }} \\
\left(\mathrm{m}^{2} / \mathrm{s}\right)\end{array}$ & $\mathrm{RC}$ \\
\hline 40 & $4,09 \times 10^{-10}$ & 4,86 & $3,46 \times 10^{-10}$ & 4,69 \\
\hline 60 & $3,75 \times 10^{-10}$ & 5,36 & $3,58 \times 10^{-10}$ & 6,32 \\
\hline 80 & $4,79 \times 10^{-10}$ & 5,28 & $4,59 \times 10^{-10}$ & 4,83 \\
\hline
\end{tabular}

La energia de activación fue determinada a partir de la pendiente de la ley de Arrhenius (ec. 5), obtenida del grafico Ln $D_{\text {ef }}$ Vs. 1/T (Fig. 3), de los datos experimentales presentados en la Tabla 1. Los valores obtenidos fueron de $3.50 \mathrm{~kJ} / \mathrm{mol}$ para cilindros y $6.34 \mathrm{~kJ} / \mathrm{mol}$ para placa. Dado que existe un quiebre de la curva en las muestras de $60^{\circ} \mathrm{C}$, es decir no hay relación lineal con la temperatura, los valores de la Ea se recalcularon para cada segmento de las curvas. Así, para cilindros se obtuvieron valores de $-3,68$ y $11,933,50 \mathrm{~kJ} / \mathrm{mol}$, para las temperaturas de 40 a $60^{\circ} \mathrm{C}$ y 60 a $80^{\circ} \mathrm{C}$, respectivamente; y para placas, valores de 1,45 y $12,08 \mathrm{~kJ} / \mathrm{mol}$, en los mismos intervalos de temperaturas. Con estos valores, que termodinámicamente representan la energía que necesitan las moléculas de agua para su migración o movimiento dentro del producto (Singh y Gupta, 2007), se puede observar cómo en ambas geometrías la mayor energía requerida para la rehidratación fue en las muestras entre las mayores temperaturas, lo que refleja un cambio más drástico en la porosidad de la muestra secada a $80^{\circ} \mathrm{C}$, que también presenta los menores coeficientes de difusión.

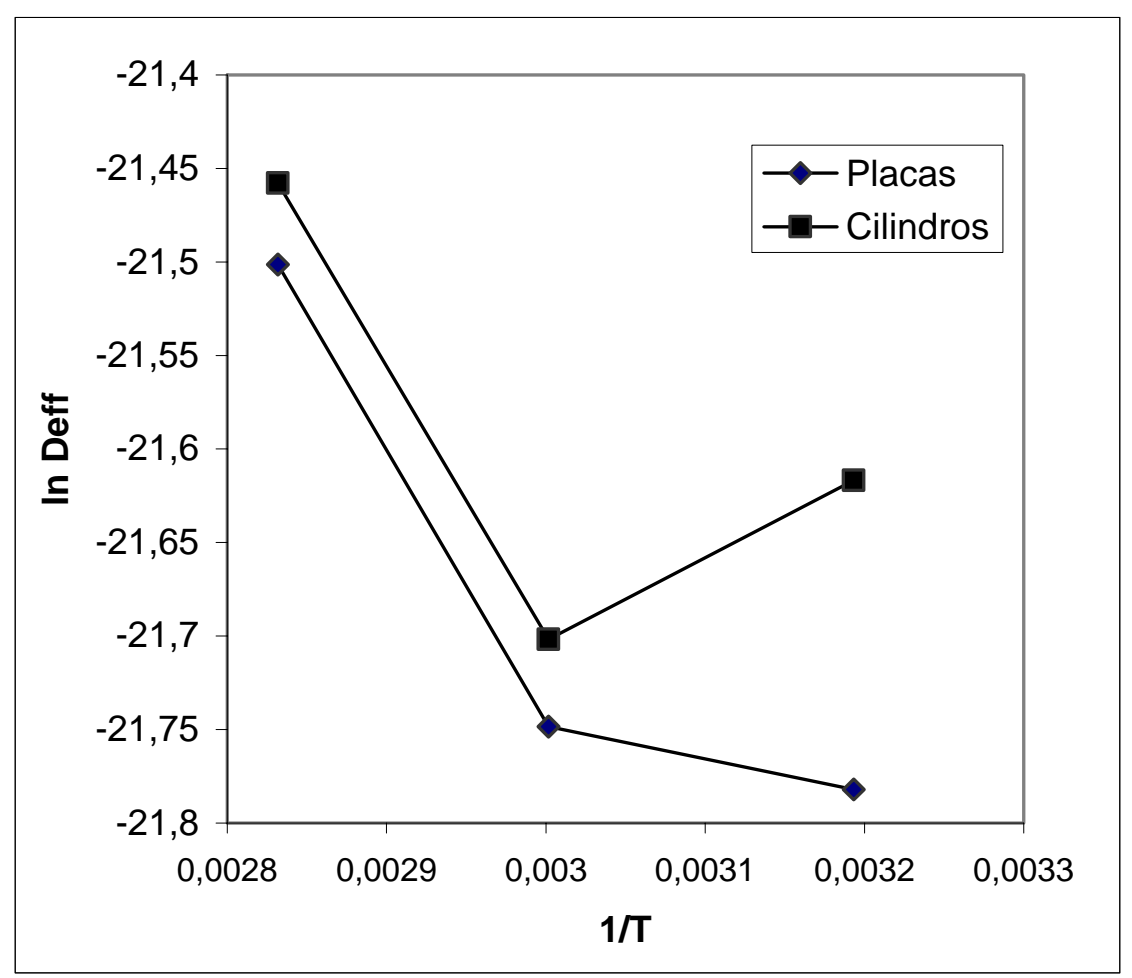

Fig. 3 Influencia de la temperatura en los coeficientes de difusión.

Se puede observar que los coeficientes de rehidratación fueron mayores en la geometría cilíndrica, puesto que cuentan con más vacíos intercelulares bien definidos (McMinn y Magee, 1997); sin embargo, dado que los coeficientes de difusión no siguen un comportamiento lineal con la 
temperatura, se observa que el proceso de rehidratación se ve influenciado principalmente por las variables de proceso utilizados durante el secado, y no necesariamente por el pretatamiento.

\section{CONCLUSIONES}

El pretratamiento facilito la pérdida de agua durante el secado, reduciendose el tiempo de proceso con la temperatura. Sin embargo, se presentó mayor daño estructural a la menor y mayor temperatura, lo que finalmente afecto la capacidad de rehidratación. Los datos experimentales mostrados demuestran que la cinética de rehidratación no guarda una relación lineal con la temperatura, debido a los cambios que sufren los poros del alimento y la gelatinización que se presenta durante el pretratamiento, además se observó que la geometría tiene gran influencia sobre los coeficientes de difusión ya que los cilindros presentaron los valores más altos.

Aunque la rehidratación y el secado son procesos que se espera que se lleven a cabo de manera similar, pero inversamente respecto al tiempo, cada una presenta una cinética diferente, debido a que la zanahoria deshidratada presenta destrucción de enlaces moleculares que provocaron una estructura contraída, por lo que al rehidratarse se forman nuevos enlaces internos, lo que finalmente influye en la capacidad de rehidratación y coeficiente de difusión.

\section{NOMENCLATURA}

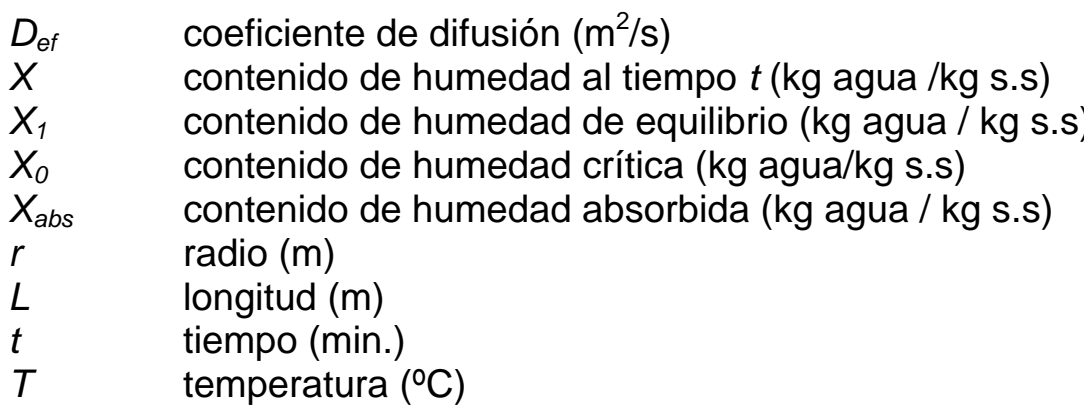

\section{REFERENCIAS}

AOAC Official method of analysis. $15^{\mathrm{a}}$ ed., Association of Official Analytical Chemists. Washington, DC (1990).

Bilbao-Saínz, C., A. Andrés y P. Fito; Hydration Kinetics of Dried Apple as Affected by Drying Conditions, Journal of Food Engineering: 68 (3), 369-376 (2005).

Cunningham, S.E., W.A.M. Mcminn, T.R.A. Magee y P.S. Richardson; Effect of Processing Conditions on the Water Absorption and Texture Kinetics of Potato. Journal of Food Engineering 84 214-223 (2008).

Crank, J.; The mathematics of diffusion. 2a ed. Oxford, UK: Clarendon Press, 1975.

Doymaz, I.; Influence of Blanching and Slice Tthickness on Drying Characteristics of Leek Slices. Chemical Engineering and Processing: 47, 41-47 (2008).

Fernández, S.M.T.; Estudio de dos Factores de Daño Celular Presentes Durante el Secado de Esporas de Trichoderma Harziainum, UNAM Instituto de Biotecnología (2006).

García-Pascual P., y otros cuatro autores; Rehydration Process of Boletus edulis mushroom: Characteristics and Modelling. Journal of the Science of Food and Agriculture: 85,1397-1404 (2005).

Geankoplis C.; Procesos de Transporte y Operaciones Unitarias, $3^{\text {a }}$ edición, 450-473, CECSA, México (1998). 
Górnicki, K. y A. Kaleta; Drying Curve Modelling of Blanched Carrot Cubes Under Natural Convection Condition, Journal of Food Engineering: 82 (2), 160-170 (2007).

Gowen, A., N. Abu-Ghannam y J. Oliveira; Influence of Pre-blanching on the Water Absorption Kinetics of Soybeans, Journal of Food Engineering: 78 (3), 965-971 (2007).

Krarup, C. e I. Moreira; Hortalizas de estación fría. Biología y Diversidad Cultural (en línea), 1998. Universidad Católica de Chile, Facultad de Agronomía e Ingeniería Forestal, Santiago. http://www.puc.cl/sw educ/hort0498. Acceso: noviembre (2007).

Krokida, M.K. y D. Marinos-Kouris; Rehydration Kinetics of Dehydrated Products, Journal of Food Engineering: 57 (1), 1-7 (2003).

Lewicki, P.P., D. Witrowa-Rajchert y J. Mariak; Changes of Structure During Rehydration of Dried Apples, Journal of Food Engineering: 32 (4), 347-350 (1997).

Marin B.E., M.R. Lemus, M.V. Flores y G.A. Vega; La Rehidratación de Alimentos Deshidratados, Revista Chilena de Nutrición: 33 (3), 527-538 (2006).

Martínez-Navarrete, N.; Termodinámica y Cinética de Sistemas Alimento Entorno, pp. 375, Servicio de publicaciones, Universidad Politécnica de Valencia, España (1998).

Marabi, A. y S. Saguy; Effect of Porosity on Rehydration of Dry Food Particulates, Journal of the Science of Food and Agriculture: 84 (10), 1105-1110 (2004).

Mayor L. y A. M. Sereno; Modelling Shrinkage During Convective Drying of Food Materials: A Review, Journal of Food Engineering: 61(3), 373-386 (2004).

McMinn, W.A.M. y T.R.A. Magee; Physical Characteristics of Dehydrated Potatoes-Part II. Journal of Food Engineering: 33 (1-2) 49-55 (1997).

Ramos, I., T. Brandão y C. Silva; Structural Changes During Air Drying of Fruits and Vegetables, Food Science and Technology International: 9, 201-206 (2003).

Singh, B. y A.K. Gupta; Mass Transfer Kinetics and Determination of Effective Diffusivity During Convective Dehydration of Pre-osmosed Carrot Cubes, Journal of Food Engineering: 79 (2), $459-470$ (2007).

Sanjuán, N., S. Simal, J. Bon y A. Mulet; Modelling of Broccoli Stems Rehydration Process, Journal of Food Engineering: 42 (1), 27-31 (1999). 